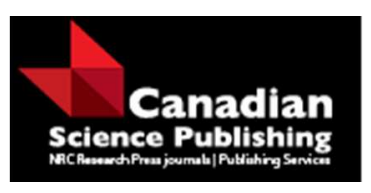

Canadian Journal of Physics

Revue canadienne de physique

\title{
High-resolution spectroscopy of giant stars in the open clusters IC 4651 and IC 4725
}

\begin{tabular}{|r|l|}
\hline Journal: & Canadian Journal of Physics \\
\hline Manuscript ID & cjp-2016-0912.R2 \\
\hline Manuscript Type: & Article \\
\hline Date Submitted by the Author: & 27-Apr-2017 \\
\hline Complete List of Authors: & $\begin{array}{l}\text { Katime Santrich, Orlando J.; Universidade de Sao Paulo Instituto de } \\
\text { Astronomia Geofisica e Ciencias Atmosfericas, Departamento de } \\
\text { Astronomia } \\
\text { Rossi, Silvia; Universidade de Sao Paulo Instituto de Astronomia Geofisica } \\
\text { e Ciencias Atmosfericas, Departamento de Astronomia }\end{array}$ \\
\hline $\begin{array}{r}\text { Keyword: } \\
\text { Is the invited manuscript for } \\
\text { consideration in a Special } \\
\text { Issue? : }\end{array}$ & $\begin{array}{l}\text { Stars: evolution, Stars: abundances, Stars: open clusters and associations, } \\
\text { Asondamental parameters, Stars: atmospheres }\end{array}$ \\
\hline & \\
\hline
\end{tabular}




\title{
High-resolution spectroscopy of giant stars in the open clusters IC 4651 and IC 4725
}

\author{
Orlando J. Katime Santrich and Silvia Rossi
}

\begin{abstract}
Open clusters are important astrophysical laboratories to study the stellar formation and evolution and to verify the disk structure of the Milky Way. We present calculations of stellar atmospheric parameters and s-process abundances for 9 giant stars in the galactic open clusters IC 4651 \& IC 4725 . These objects have their memberships confirmed from dynamic studies and chemical analysis. The high resolution spectra are available in the FEROS ESO archive. We have applied an line by line analysis relative to Juno solar spectrum to determine the stellar atmospheric parameters and chemical abundances of Y II, Zr I, La II, Ce II and Nd II under LTE-hypothesis. The obtained results were compared to the literature values. The derived s-process abundance pattern agrees with the most recent behaviors reported for giant stars in galactic open clusters.

Resume francais:
\end{abstract}

\section{Introduction}

Open clusters (OC) can be seen as important astrophysical objects to study stellar formation and evolution, and galactic structure and evolution. These objects help us to understand how conceivable is the simultaneous formation and evolution of stars from the same molecular cloud. They can also be thought as laboratories to study the stellar interiors because chemical abundance studies allow to probe usual assumptions made about mixing phenomena at RGB (Red Giant Branch) and AGB (Asymptotic Giant Branch) stellar evolutionary phases. One of the most promising ways to analyze the structure of the Milky Way (MW), specifically its disk, is studying OCs since their radial metallicity gradients outline the disk structure and reveal the distinction between thin and thick disk. To unveil the formation of the Galaxy is crucial to analyze the galactic thin disk as it is the component with higher stellar formation rate and the easiest to be observed.

Calculations of heavy element abundances have become more and more important in recent years. There are only a few stars in OC with such measured abundances since high signal-to-noise $(\mathrm{S} / \mathrm{N})$ spectra are necessary to identify the absorption lines of those elements. Besides that, the existence of strong molecular opacity make hard tangle these calculations such as it happens in the spectra of AGB stars. Furthermore, abundances of s-process elements in RGB cluster stars are interesting to find changes in the stellar evolution, as found in Barium Stars due to mass transfer events [1].

In this work we present abundances of heavy elements for two galactic OC, IC 4651 and IC 4725. These clusters are the first analyzed within our project on the behavior of the heavy elements in the galactic disk. IC 4651 was studied by many different authors [2], [3], [4], [5] \& [6], but no s-process element abundances were determined. In addition, there are three stars in common with the work of [7] which present abundances of many chemical elements. IC 4725 has two stars in common with [8]. Whenever possible, we compare our results with those obtained in the literarure.

Received. Accepted

Orlando J. Katime Santrich and Silvia Rossi. Universidade de São Paulo, Instituto de Astronomia, Geofísica e Ciências atmosféricas, Departamento de Astronomia, Rua do Matão 1226, 05508-090 São Paulo/SP, Brazil 


\section{Methodology}

Data for IC 4651 and IC 4725 appear in many different sources. Information on distance, reddening, distance modulus and age were gathered from WEBDA ${ }^{1}$ website and are presented in Table 1.

The high resolution spectra $(\mathrm{R}=48000)$ were obtained from FEROS ESO archive. Stars have their memberships confirmed from dynamic studies [9]. The stars analyzed in this work are shown in Table 2. For all stars in OC IC 4651 the exposure times were $1800 \mathrm{~s}$, while for IC 4725-150 and IC 4725-251 exposure times were $90 \mathrm{~s}$ and $60 \mathrm{~s}$, respectively. For all stars, the spectra have the $(\mathrm{S} / \mathrm{N})$ around 200 and for the Juno solar spectrum is 800 .

Radial velocities for stars considered in this work were obtained from Doppler shift and are shown in Table 2. Note that exists a difference of almost $5 \mathbf{k m ~ s}^{-1}$ between our $V_{r}^{a}$ and $V_{r}^{b}$ for the star IC 4651-14527. However, our adopted value $\left(V_{r}^{a}=-27.16 \pm 0.96\right)$ is very similar to the value found by [10] of $-27.88 \mathrm{Km} \mathrm{s}^{-1}$. The spectrum from [10] was observed in $04 / 29 / 2000$ while our was obtained in $06 / 29 / 2010$, according to the ESO/archive. In the Mermilliod's survey, IC 4651-14527 was observed between $03 / 08 / 1983$ and $07 / 10 / 1996$ and is not reported as binary. Therefore, this star has a very low probability to be binary and, if so, its orbit would be very eccentric.

Stellar atmospheric parameters and s-process abundances were calculated based on LTE-hypothesis: effective temperatures were obtained from excitation equilibrium, microturbulence velocities from zero slope between Fe I abundances and reduced equivalent width, surface gravities from ionization equilibrium between $\mathrm{Fe} \mathrm{I}$ and $\mathrm{Fe}$ II lines, and the metallicity $[\mathrm{Fe} / \mathrm{H}]$ is established from the final value fixed by the ionization equilibrium. Because of our sample has stellar parameters published in the literature, these values were used as initial guesses in the process. For the stars IC 4651-6333 and -9025 with no previous spectroscopic analysis, the mean values for $\mathrm{T}_{e f f}, \log \mathrm{g}, \xi$ and $[\mathrm{Fe} / \mathrm{H}]$ of the cluster IC 4651 were used as initial values in the calculations.

In this type of spectroscopic analysis is always necessary to use the Sun as the reference star, for both dwarf and giant stars. In some cases, giants are compared to the Arcturus giant but it is very frequent to transform the Arcturus abundances in solar. In this work, to obtain stellar parameters and s-process abundances with the best possible precision, we have decided to use a line-by-line analysis (lbl analysis) relative to $\mathrm{Juno}^{2}$ solar spectrum. This method allows to get abundances by means of the relationship: $\delta \mathbf{A}_{i}=\mathbf{A}_{i}^{\star}-\mathbf{A}_{i}^{\odot}$, where $\mathbf{A}_{i}^{\star}$ is the stellar logarithmic abundance of a chemical species $i$ for a given wavelength $\lambda$, and $\mathbf{A}_{i}^{\odot}$ is the same related to the Sun. Accordingly $\delta \mathbf{A}_{i}$ represents the differential abundance of the species $i$ for the same $\lambda$.

This method has been used for several purposes in the literature as to study stellar evolution effects [11] and [12], to find planetary signatures in host-stars [13], [14] and [15], to calculate chemical abundances in the solar neighborhood [16] and [17], to find abundance anomalies in stellar clusters [18] and to distinct populations in the stellar halo [19] and [20]. [21] ${ }^{3}$ show a comparison between the classical and differential abundance estimates.

To carry out the analysis we have used the python-code $\mathrm{q}^{2}$ (qoyllur-quipu ${ }^{4}$ ). This code uses the Kurucz atmospheric grids ${ }^{5}$ and spectral code MOOG $^{6}$. The absorption lines of the Iron I \& II were taken from [23] and [24] and are shown in Table 7. The lines of the s-process elements were taken from several sources and are shown in Table 8.

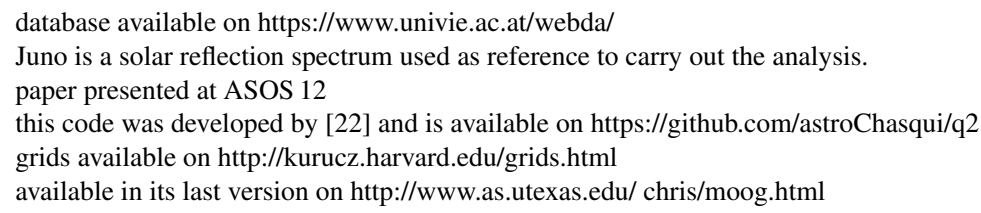




\section{Results and analysis}

The open cluster IC 4651 was previously studied in the literature using high-resolution spectroscopy. The stellar atmospheric parameters for the stars IC 4651-7646 and -9122 were calculated by [5] while [7] calculated the chemical abundances for the stars IC 4651-8540, -9791 and - 14527 using the stellar parameters calculated by [10]. The stars IC $4651-6333 \&-9025$ have no previous determination of chemical abundances, and therefore, it is the first time they are spectroscopically studied. In the case of IC 4725, [8] has also analyzed the stars IC 4725-150 \& -251 . This OC has no other stars chemically studied.

Such as can be observed in Table 3 , the results indicate that the studied sample has effective temperatures and surface gravities typically of cool giant stars. The metallicities are slightly enhanced in IC 4651-7646, IC 4651-9025, IC 4651-14527 and IC 4725-150 while for IC 4651-8540, IC 4651-9791 and IC 4725-251 are slightly lower than the solar value. IC 4651-6333 and IC 4651-9122 have solar metallicities.

Chemical abundances for the s-process elements are shown in Table 4. From these results we have noted that there is a small enhancement of La II and Nd II in some stars when compared with the field giants, showed in Figure 1 left. Those elements have a tendency to be lightly increased in the two OC. However, this enhancement is not large enough to considere these cluster giants as stars with sprocess overabundances such as it happens with the Barium stars discovered in the OC NGC 5822 [1]. Furthermore, this figure also shows that $[\mathrm{s} / \mathrm{Fe}]$ ratios found in these cluster giants are similar to the ones in the field. The Figure 1 right presents a comparison between our results and other cluster giants reported in the literature. These OC were divided by age using the criteria of [25]: old (age > 1.0 Gyr) and young (age $<1.0 \mathrm{Gyr}$ ) OC. This separation is important to unveil the differences between the thin and thick galactic disks because the old OC can offer a view of what could be happening on the thick disk. Old and young OC can also be important to ascertain the differences in abundances, because if there are, they could be due to nuclear processes in intermediate-mass stars, such like that in globular clusters [26]. The literature data were taken from [26]; [27]; [28]; [29]; [30]; [31]; [32]; [33]; [34]; [35]; [36]; [37]; [38]; [39] and [40]. It has to be mentioned that IC 4651 and IC 4725 have average s-process element abundances in agreement with other cluster giants in literature.
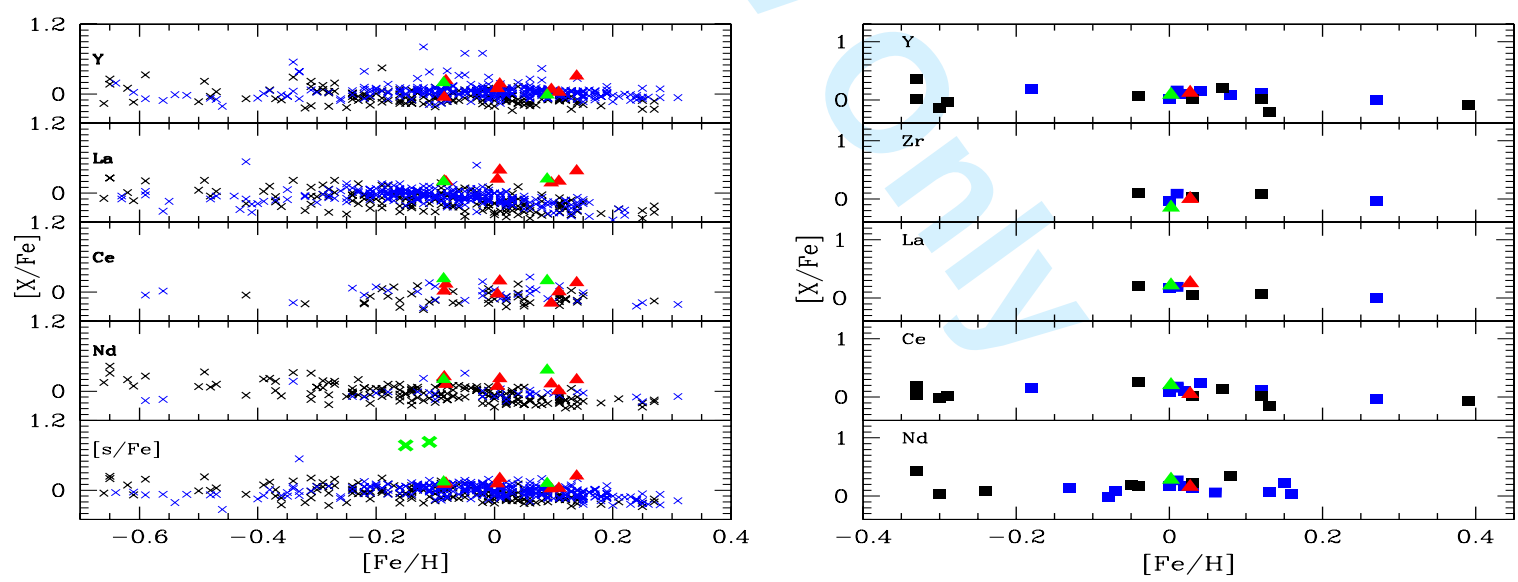

Fig. 1. Left: s-process abundances star by star. Black crosses: field giants of [41]; blue crosses: field giants [42]; green squares: barium stars of [1]; green triangles: giants in IC 4725; red triangles: giants in IC 4651. Right: sprocess abundances by cluster. Black squares: old OC from literature; blue squares: young OC from literature; green square: IC 4725; red square: IC 4651. 
In Table 5 are shown the stellar parameters calculated by [10] and s-process abundances calculated by [7] for the stars IC 4651-9791, -8540 and -14527 . We can see that there are more differences than similarities between our results and theirs. This may be due to differences in the way of analysis. [10], for instance, did automatic measurements of equivalent widths using the spectrum analysis package developed in Padova [43] and [44], while [7] used Carretta's ([10]) stellar parameters to calculate their chemical abundances. In the case of IC 4725, the differences should be on the log gf values used by [8]. The authors [8], [10] and [7] used different line lists and none of them performed the lbl analysis as done in this work.

In order to apply the $\mathrm{lbl}$ analysis is necessary that the objects and the standard star have similar spectral types ( $\mathrm{F}, \mathrm{G}$ or $\mathrm{K}$ in this case). This is observed in the Table 2 where six stars in the two OC have spectral types G \& K confirmed. Another constrain is that the absorption lines are formed in different atmospheric regions in giants and dwarfs. Then, this analysis among them are limited by the number of lines, specially when the s-process lines are considered. However, if the solar spectrum has high $(\mathrm{S} / \mathrm{N})$ is possible to obtain high precision abundances, even with few lines available. To show this, we compare non-differential with differential Nd II abundances for the star IC 4651-14527, see Table 6. Then, in the non-differential we obtained the mean abundance ratios of $[\mathrm{Nd} \mathrm{II} / \mathrm{H}]_{1}=0.631 \pm 0.479 \mathrm{dex},[\mathrm{Nd} \mathrm{II} / \mathrm{H}]_{2}=0.795 \pm 0.458 \mathrm{dex}$ and $[\mathrm{Nd} \mathrm{II} / \mathrm{H}]_{1}=0.678 \pm 0.519 \mathrm{dex}$ by using the solar abundance of $\log \mathrm{Nd} \mathrm{II}=1.42 \mathrm{dex}[45]$ and the mean values of $(\log \mathrm{Nd} \mathrm{II})_{1}=2.051 \mathrm{dex}$; $(\log \mathrm{Nd} \text { II })_{2}=2.215 \mathrm{dex}$ and $(\log \mathrm{Nd} \mathrm{II})_{3}=2.098 \mathrm{dex}$ respectively. While for $\mathrm{lbl}$ analysis we obtained the mean abundance ratio of $\delta[\mathrm{Nd} \mathrm{II} / \mathrm{H}]=0.168 \pm 0.230 \mathrm{dex}$. This last value is in better agreement with those expected to RGB stars.

\section{Conclusions}

Because of important differences reported in the literature, the differential method is the best option to eliminate the dependency on the log gf values that chemical abundance calculations present. In this work, the line-by-line analysis relative to Juno solar spectrum was an excellent option to avoid long and subjetive comparations between the different line lists published in literarure. This situation is specially shown for the Nd II abundances in the star IC 4651-14527. From its atmospheric parameters we see that this star is not in AGB-phase and, as mentioned above, it has a very low probability of being binary. Then it is very unlikely that this star has overabundances of the s-process (Nd II in this case) as shown by the abundance ratios $[\mathrm{Nd} \mathrm{II} / \mathrm{H}]_{1}=0.631 \pm 0.479 \mathrm{dex},[\mathrm{Nd} \mathrm{II} / \mathrm{H}]_{2}=0.795 \pm 0.458 \mathrm{dex}$ and $[\mathrm{Nd} \mathrm{II} / \mathrm{H}]_{1}=0.678 \pm 0.519$ dex. Therefore the value $\delta[\mathrm{Nd} \mathrm{II} / \mathrm{H}]=0.168 \pm 0.230 \mathrm{dex}$ obtained from the $\mathrm{lbl}$ analysis is the most plausible in this case.

For the first time the stars IC 4651-6333 and -9025 were studied, since no previous abundance results were found in the literature. Their stellar parameters and s-process abundances are similar to the other giants within IC 4651. We obtained abundances for the chemical elements La II and Ce II in IC 4725. The stars IC 4725-150 \&-251 have no previous results for these elements.

The stars analyzed in this work show stellar parameters in accordance to RGB stars and chemical abundances similar to other cluster giants of the galatic disk also studied using high-resolution. In other words, our results are congruent with the global chemical evolution of the MW. This is an ongoing project and more stars in different $\mathrm{OC}$ will be analysed in the future.

\section{Acknowledgment}

O. J. Katime Santrich and S. Rossi would like to acknowledge to CAPES, FAPESP and CNPQ for the financial support.

Based on data obtained from the ESO Science Archive Facility under request numbers 216546 and 230944 and username ojksantrich. 


\section{References}

1. O. J. Katime Santrich, C. B. Pereira, \& D. B. de Castro. AJ, 146, 39K, (2013).

2. S. Randich, F. Primas, L. Pasquini, \& R. Pallavicini. A\&A, 387, 222R, (2002).

3. L. Pasquini, S. Randich, M. Zoccali, V. Hill, C. Charbonnel, \& B. Nordstrom. A\&A, 424, 951P, (2004).

4. K. Biazzo, L. Pasquini, L. Girardi, A. Frasca, L. da Silva, J. Setiawan, E. Marilli, A. P. Hatzes \& S. Catalano. A\&A, 475, 981B, (2007).

5. N. C. Santos, C. Lovis, G. Pace, J. Meléndez \& D. Naef. A\&A, 493, 309S, (2009).

6. R. Smiljanic, L. Pasquini, C. Charbonnel \& N. Lagarde. A\&A, 510A, 50S, (2010).

7. S. Mikolaitis, G. TautvaiSiené, R. Gratton, A. Bragaglia \& E. Carretta. MNRAS, 413, 2199M, (2010).

8. R.E. Luck. ApJS, 91, 309L, (1994).

9. J. C. Mermilliod, M. Mayor \& S. Udry. A\&A, 485, 303M, (2008).

10. E. Carretta, A. Bragaglia, R. G. Gratton, \& M. Tosi. A\&A, 422, 951C, (2004).

11. T. R. Monroe, J. Meléndez, I. Ramírez, et al. ApJ, 774L, 32M, (2013).

12. M. Tucci Maia, J. Meléndez, M. Castro, M. Asplund, et al. A\&A, 576L, 10T, (2015).

13. J. Meléndez, M. Asplund, B. Gustafsson \& D. Yong. AJ, L66, 704, (2009).

14. I. Ramírez, J. Meléndez \& M. Asplund. A\&A, L17, 508, (2009).

15. S. C. Schuler, Z. A. Vaz, O. J. Katime Santrich, et al. ApJ, 815, 5S, (2015).

16. P.E. Nissen. A\&A, A52, 579, (2015).

17. H. Reggiani, J. Meléndez. et al. A\&A, A67, 586, (2016).

18. D. Yong, J. Meléndez, F. Grundahl, et al. MNRAS, 434, 3542Y, (2013).

19. I. Ramírez, J. Meléndez \& J. Chanamé. ApJ, 757, 164, (2012).

20. C K. Fishlock, D. Yong, et al. MNRAS, 57F, (2017).

21. H. Reggiani \& J. Meléndez. Accepted in CaJP/ASOS-12, (2017).

22. I. Ramirez, J. Meléndez \& M. Asplund. A\&A, 561, A7, (2014).

23. D. L. Lambert, J. E. Heath, M. Lemke, M \& J. Drake. ApJS, 103, 183, (1996).

24. S. Castro, R. M. Rich, M. Grenon, B. Barbuy \& J. K. McCarthy. AJ, 114, 376, (1997).

25. E. D. Friel. ARA\&A, 313, 381F, (1995).

26. E. D. Friel, H. R. Jacobson \& C. A. Pilachowski. AJ, 139, 1942F, (2010).

27. R. G. Gratton \& G. Contarini. A\&A, 283, 911G, (1994).

28. J. A. Brown, G. Wallerstein, D. Geisler \& J. B. Oke. AJ, 112, 1551B, (1996).

29. E. Carretta, A. Bragaglia, R. G. Gratton \& M. Tosi. A\&A, 441, 131C, (2005).

30. E. D. Friel, H. R. Jacobson \& C. A. Pilachowski. AJ, 129, 2725F, (2005).

31. D. Yong, B. W. Carney \& M. L. Teixera de Almeida. AJ, 130, 597Y, (2005).

32. R. Smiljanic, R. Gauderon, P. North, B. Barbuy, C. Charbonnel \& N. Mowlavi. A\&A, 502, 267S, (2009).

33. S. Villanova, G. Carraro \& I. Saviane, I. A\&A, 504, 845V, (2009).

34. E. Pancino, R. Carrera, E. Rossetti \& C. Gallart. A\&A, 511A, 56P, (2010).

35. H. R. Jacobson, E. D. Friel, \& C. A. Pilachowski. AJ, 135, 2341J, (2008).

36. H. R. Jacobson, E. D. Friel, \& C. A. Pilachowski. AJ, 141, 58J, (2011).

37. R. Carrera \& E. Pancino. A\&A, 535A, 30C, (2011).

38. E. Maiorca, S. Randich, M. Busso, L. Magrini \& S. Palmerini. ApJ, 736, 120M, (2011).

39. O. J. K. Santrich, C. B. Pereira \& N. A. Drake. A\&A, 554A, 2S, (2013).

40. J. V. Sales Silva, V. J. Peña Suárez, O. J. Katime Santrich, C. B. Pereira, N. A. Drake \& F. Roig. AJ, 148, 83S, (2014).

41. T. V. Mishenina, O. Bienayme, T. I. Gorbaneva, C. Charbonnel, C. Soubiran, S. A. Korotin, \& V. V. Kovtyukh. A\&A, 456, 1109M, (2006).

42. R. E. Luck \& U. Heiter. AJ, 133, 2464L, (2007).

43. A. Bragaglia, E. Carretta, R. G. Gratton, et al. AJ, 121, 327, (2001).

44. E. Carretta, R. Gratton, J. G.Cohen, T. C. Beers \& N. Christlieb. AJ, 124, 481C, (2002).

45. M. Asplund, N. Grevesse, A. J. Sauval \& P. Scott. ARA\&A, 47, 481A, (2009).

46. H. Van Winckel \& M. Reyniers. A\&A, 345, 135, (2000).

47. E. A. Den Hartog, J. E. Lawler, C. Sneden \& J. J. Cowan. ApJS, 148, 543D, (2003).

48. B. Edvardsson, J. Andersen, B. Gustafsson, D. L. Lambert, P. E. Nissen \& J. Tomkin. A\&A, 275, 101E, 
Table 1. Basic parameters for the open clusters analyzed in this paper.

\begin{tabular}{l|cc}
\hline \hline Parameter & IC 4651 & IC 4725 \\
\hline \hline Right ascension $\alpha(2000)$ & 172449 & 183147 \\
Declination $\delta(2000)$ & -495600 & -190700 \\
Galactic longitude & 340.09 & 13.70 \\
Galactic latitude & -7.91 & -4.43 \\
Distance [pc] & 888 & 620 \\
Reddening [mag] & 0.12 & 0.48 \\
Distance modulus [mag] & 10.10 & 10.44 \\
log Age & 9.06 & 7.97 \\
\hline \hline
\end{tabular}

Note. Information obtained from WEBDA database: http://www.univie.ac.at/webda/

Table 2. Stars analyzed in this work.

\begin{tabular}{l|ccccccc}
\hline \hline Star & $\alpha$ & $\delta$ & $\mathrm{V}$ & $\mathrm{B}-\mathrm{V}$ & $\begin{array}{c}\mathrm{V}_{r}^{a} \\
\mathrm{Km} \mathrm{s}^{-1}\end{array}$ & $\begin{array}{c}\mathrm{V}_{r}^{b} \\
\mathrm{Km} \mathrm{s}^{-1}\end{array}$ & $\begin{array}{c}\text { Spec. type } \\
\text { (source) }\end{array}$ \\
\hline \hline IC 4651-6333 & 172433 & -495456 & 10.75 & 0.96 & $-29.56 \pm 0.79$ & $-30.87 \pm 0.10$ & - \\
IC 4651-7646 & 172442 & -495906 & 10.36 & 1.04 & $-30.65 \pm 0.96$ & $-31.18 \pm 0.13$ & K0 (WEBDA) \\
IC 4651-8540 & 172447 & -495407 & 10.89 & 1.13 & $-29.93 \pm 0.84$ & $-30.36 \pm 0.14$ & - \\
IC 4651-9025 & 172450 & -495727 & 10.90 & 1.13 & $-29.70 \pm 0.93$ & $-30.46 \pm 0.14$ & K0 (SIMBAD) \\
IC 4651-9122 & 172450 & -495656 & 10.89 & 1.22 & $-29.77 \pm 1.24$ & $-30.58 \pm 0.13$ & - \\
IC 4651-9791 & 172414 & -495307 & 10.43 & 1.33 & $-30.75 \pm 1.80$ & $-31.44 \pm 0.12$ & K2 (WEBDA) \\
IC 4651-14527 & 172524 & -495547 & 10.94 & 1.14 & $-27.16 \pm 0.96$ & $-31.85 \pm 0.26$ & K0 (SIMBAD) \\
\hline IC 4725-150 & 183213 & -190726 & 7.36 & 1.53 & $2.15 \pm 0.68$ & $2.84 \pm 0.06$ & K0 (SIMBAD) \\
IC 4725-251 & 183230 & -185822 & 6.99 & 1.35 & $3.97 \pm 1.30$ & $2.13 \pm 0.12$ & G3 (SIMBAD) \\
\hline \hline
\end{tabular}

Note 1. Where $\mathrm{V}_{r}^{a}$ and $\mathrm{V}_{r}^{b}$ are the radial velocities obtained in this work and Mermilliod's survey respectively.

Note 2. SIMBAD astronomical database is available on http://simbad.u-strasbg.fr/simbad/

(1993).

49. C. Sneden, A. McWilliam, G. W. Preston, et al. ApJ, 467, 819, (1996).

50. B. E. Reddy, J. Tomkin, D. L. Lambert, \& C. Allende Prieto. MNRAS, 340, 30, (2003).

51. L. I. Antipova, A. A. Boyarchuk, Y. V. Pakhomov \& M. V. Yushkin. ARep, 49, 535, (2005).

52. V. V. Smith, K. Cunha, A. Jorissen \& H. M. J. Boffin. A\&A, 315, 179, (1996).

53. M. Reyniers, H. Van Winckel, R. Gallino \& O. Straniero. A\&A, 417, 269, (2004). 
Table 3. Stellar parameters for the stars analyzed in this work.

\begin{tabular}{l|ccccc}
\hline Star & $\begin{array}{c}\mathrm{T}_{\text {eff }} \\
\text { Kelvins }\end{array}$ & $\begin{array}{c}\text { log g } \\
\mathrm{dex}\end{array}$ & $\begin{array}{c}\xi \\
\mathrm{Km} \mathrm{s}^{-1}\end{array}$ & $\begin{array}{c}{[\mathrm{Fe} / \mathrm{H}](\#)} \\
\mathrm{dex}\end{array}$ & $\begin{array}{c}{[\mathrm{Fe} \mathrm{II} / \mathrm{H}](\#)} \\
\mathrm{dex}\end{array}$ \\
\hline \hline IC 4651-6333 & $4647 \pm 99$ & $2.55 \pm 0.23$ & $2.0 \pm 0.1$ & $0.009 \pm 0.078(63)$ & $0.011 \pm 0.145(12)$ \\
IC 4651-7646 & $4947 \pm 31$ & $2.72 \pm 0.10$ & $1.6 \pm 0.1$ & $0.096 \pm 0.033(65)$ & $0.100 \pm 0.085(13)$ \\
IC 4651-8540 & $4865 \pm 95$ & $2.68 \pm 0.27$ & $2.0 \pm 0.2$ & $-0.085 \pm 0.086(69)$ & $-0.085 \pm 0.131(14)$ \\
IC 4651-9025 & $4906 \pm 52$ & $2.77 \pm 0.19$ & $1.7 \pm 0.1$ & $0.109 \pm 0.068(67)$ & $0.109 \pm 0.110(14)$ \\
IC 4651-9122 & $4745 \pm 55$ & $2.69 \pm 0.16$ & $1.7 \pm 0.1$ & $0.005 \pm 0.054(60)$ & $0.008 \pm 0.092(13)$ \\
IC 4651-9791 & $4564 \pm 83$ & $2.20 \pm 0.25$ & $1.9 \pm 0.1$ & $-0.082 \pm 0.082(65)$ & $-0.084 \pm 0.082(12)$ \\
IC 4651-14527 & $5015 \pm 70$ & $3.09 \pm 0.14$ & $1.8 \pm 0.1$ & $0.139 \pm 0.058(64)$ & $0.139 \pm 0.113(13)$ \\
\hline Mean values & $4813 \pm 68$ & $2.70 \pm 0.11$ & $1.8 \pm 0.1$ & $0.027 \pm 0.037$ & $0.028 \pm 0.037$ \\
\hline IC 4725-150 & $4582 \pm 57$ & $1.79 \pm 0.23$ & $2.5 \pm 0.2$ & $0.089 \pm 0.070(31$ & $0.088 \pm 0.033(7)$ \\
IC 4725-251 & $5217 \pm 51$ & $1.89 \pm 0.17$ & $4.5 \pm 0.4$ & $-0.086 \pm 0.060(48)$ & $-0.086 \pm 0.134(6)$ \\
\hline Mean Values & 4900 & 2.74 & 3.5 & 0.002 & 0.001 \\
\hline \hline
\end{tabular}

Table 4. s-process abundance ratios for IC 4651 and IC 4725.

\begin{tabular}{l|cccccc}
\hline \hline Star & {$[\mathrm{YII} / \mathrm{Fe}]$} & {$[\mathrm{ZrI} / \mathrm{Fe}]$} & {$[\mathrm{LaII} / \mathrm{Fe}]$} & {$[\mathrm{CeII} / \mathrm{Fe}]$} & {$[\mathrm{NdII} / \mathrm{Fe}]$} & {$[\mathrm{s} / \mathrm{Fe}]$} \\
\hline \hline IC 4651-6333 & $0.176 \pm 0.138(4)$ & $0.036 \pm 0.112(3)$ & $0.395 \pm 0.068(3)$ & $0.192 \pm 0.143(3)$ & $0.216 \pm 0.072(4)$ & $0.203 \pm 0.128$ \\
IC 4651-7646 & $0.079 \pm 0.053(5)$ & $-0.071 \pm 0.078(3)$ & $0.175 \pm 0.089(3)$ & $-0.187 \pm 0.151(3)$ & $0.127 \pm 0.123(5)$ & $0.025 \pm 0.150$ \\
IC 4651-8540 & $-0.055 \pm 0.081(5)$ & $0.080 \pm 0.118(3)$ & $0.209 \pm 0.076(3)$ & $0.019(1)$ & $0.250(2)$ & $0.101 \pm 0.128$ \\
IC 4651-9025 & $0.030 \pm 0.037(3)$ & $-0.089 \pm 0.096(3)$ & $0.204 \pm 0.047(3)$ & $0.005 \pm 0.058(3)$ & $0.007(2)$ & $0.031 \pm 0.107$ \\
IC 4651-9122 & $0.092 \pm 0.106(5)$ & $-0.031 \pm 0.107(3)$ & $0.238 \pm 0.059(3)$ & $-0.033 \pm 0.080(3)$ & $0.083 \pm 0.105(4)$ & $0.108 \pm 0.115$ \\
IC 4651-9791 & $0.232(2)$ & $-0.077 \pm 0.115(3)$ & $0.197 \pm 0.047(3)$ & $0.137(2)$ & $0.119 \pm 0.058(4)$ & $0.122 \pm 0.120$ \\
IC 4651-14527 & $0.309 \pm 0.078(5)$ & $0.170 \pm 0.089(3)$ & $0.381 \pm 0.057(3)$ & $0.165 \pm 0.133(3)$ & $0.029 \pm 0.230(5)$ & $0.211 \pm 0.137$ \\
\hline Mean value & $0.123 \pm 0.152$ & $0.003 \pm 0.119$ & $0.257 \pm 0.112$ & $0.052 \pm 0.158$ & $0.137 \pm 0.115$ & $0.114 \pm 0.073$ \\
\hline IC 4725-150 & $-0.017 \pm 0.159(4)$ & $-0.199 \pm 0.084(3)$ & $0.243(2)$ & $0.199(2)$ & $0.362 \pm 0.238(4)$ & $0.118 \pm 0.224$ \\
IC 4725-251 & $0.195(2)$ & $-0.095(1)$ & $0.195(1)$ & $0.213 \pm 0.198(3)$ & $0.202 \pm 0.172(6)$ & $0.142 \pm 0.133$ \\
\hline Mean value & 0.089 & -0.147 & 0.219 & 0.206 & 0.282 & 0.130 \\
\hline \hline
\end{tabular}

Table 5. Stellar parameters and s-process abundance ratios found in literature in commom with our sample.

\begin{tabular}{l|ccc|ccccc}
\hline OC & $\mathrm{T}_{e f f}$ & $\log \mathrm{g}$ & {$[\mathrm{Fe} / \mathrm{H}]$} & {$[\mathrm{YII} / \mathrm{Fe}]$} & {$[\mathrm{ZrI} / \mathrm{Fe}]$} & {$[\mathrm{LaII} / \mathrm{Fe}]$} & {$[\mathrm{CeII} / \mathrm{Fe}]$} & {$[\mathrm{NdII} / \mathrm{Fe}]$} \\
\hline \hline IC 4651-9791/72 & 4500 & 2.23 & 0.13 & $-0.03 \pm 0.04(6)$ & $-0.13(2)$ & $-0.03(2)$ & $0.07(2)$ & $0.15 \pm 0.06(3)$ \\
IC 4651-8540/76 & 4620 & 2.26 & 0.11 & $-0.07 \pm 0.01(6)$ & $-0.07(2)$ & $0.04(2)$ & $0.13(2)$ & $0.04 \pm 0.05(3)$ \\
IC 4651-14527/146 & 4730 & 2.14 & 0.10 & $0.04 \pm 0.04(6)$ & $-0.10(2)$ & $0.05(2)$ & $0.10(2)$ & $0.08 \pm 0.03(3)$ \\
\hline IC 4725-150/HD 170820 & 4700 & 1.63 & 0.22 & - & $-0.06 \pm 0.05(3)$ & - & - & $-0.03(1)$ \\
IC 4725-251/HD 170885 & 5100 & 1.00 & 0.08 & 0.13 & $0.05 \pm 0.16(3)$ & - & - & $0.49(2)$ \\
\hline \hline
\end{tabular}




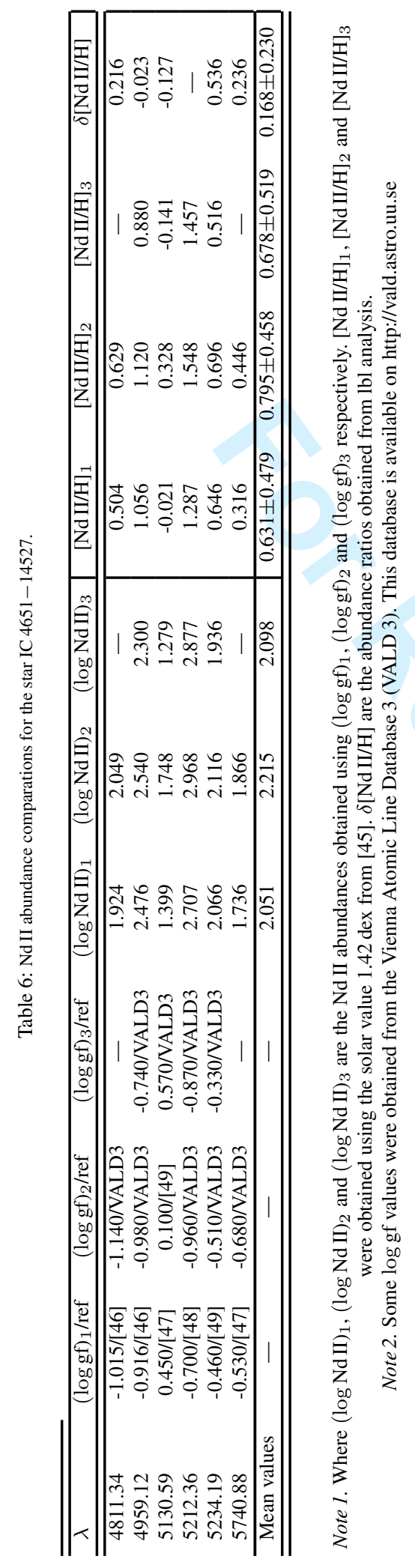




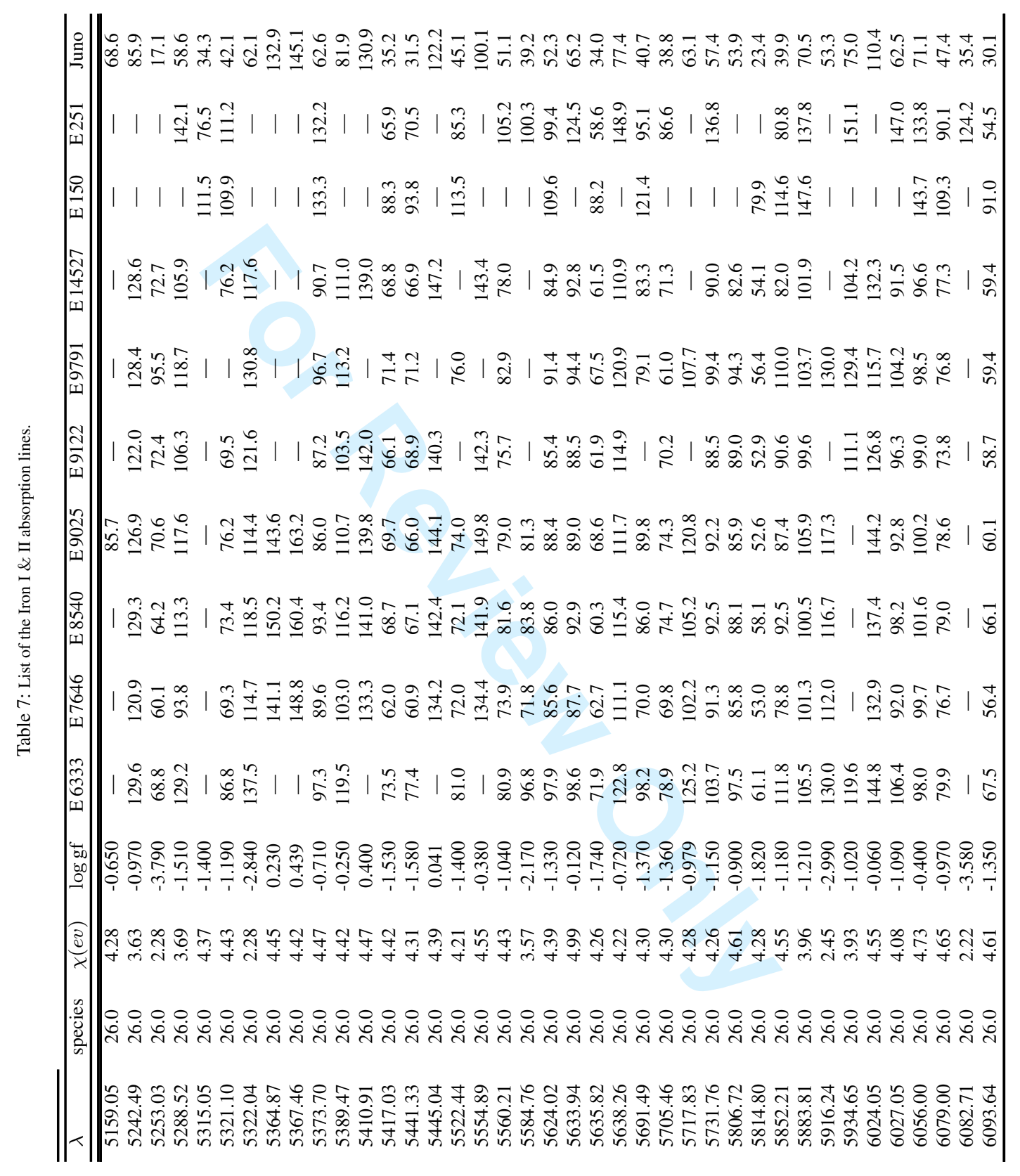

NRC Canada 


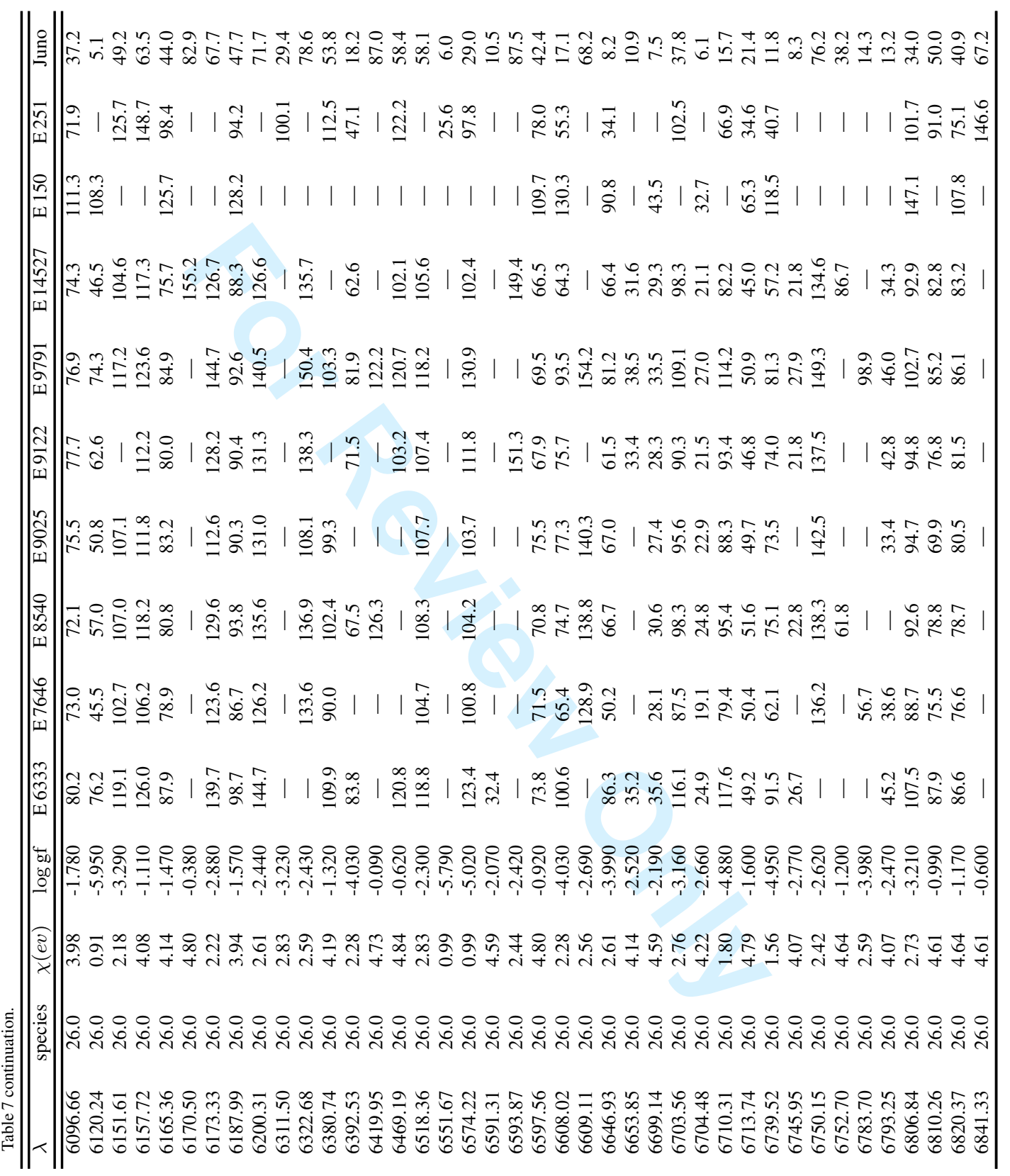




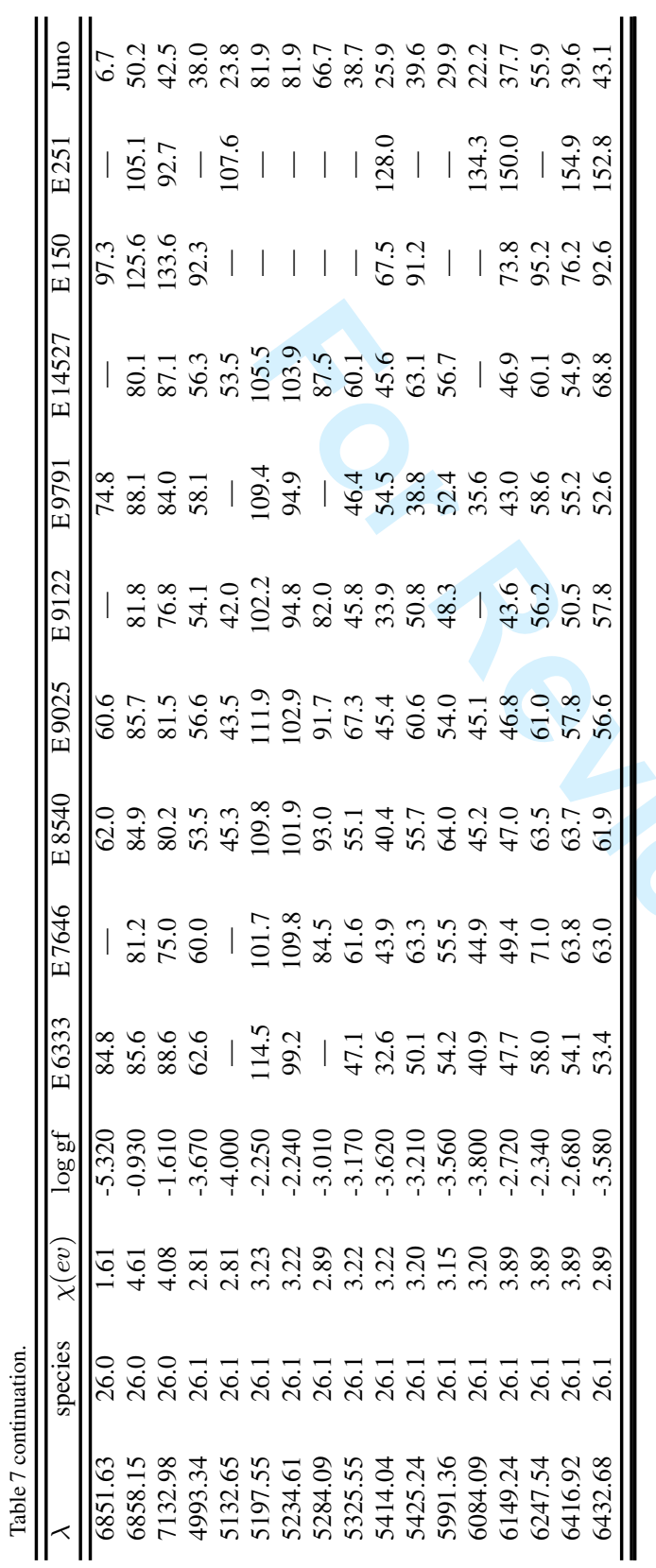




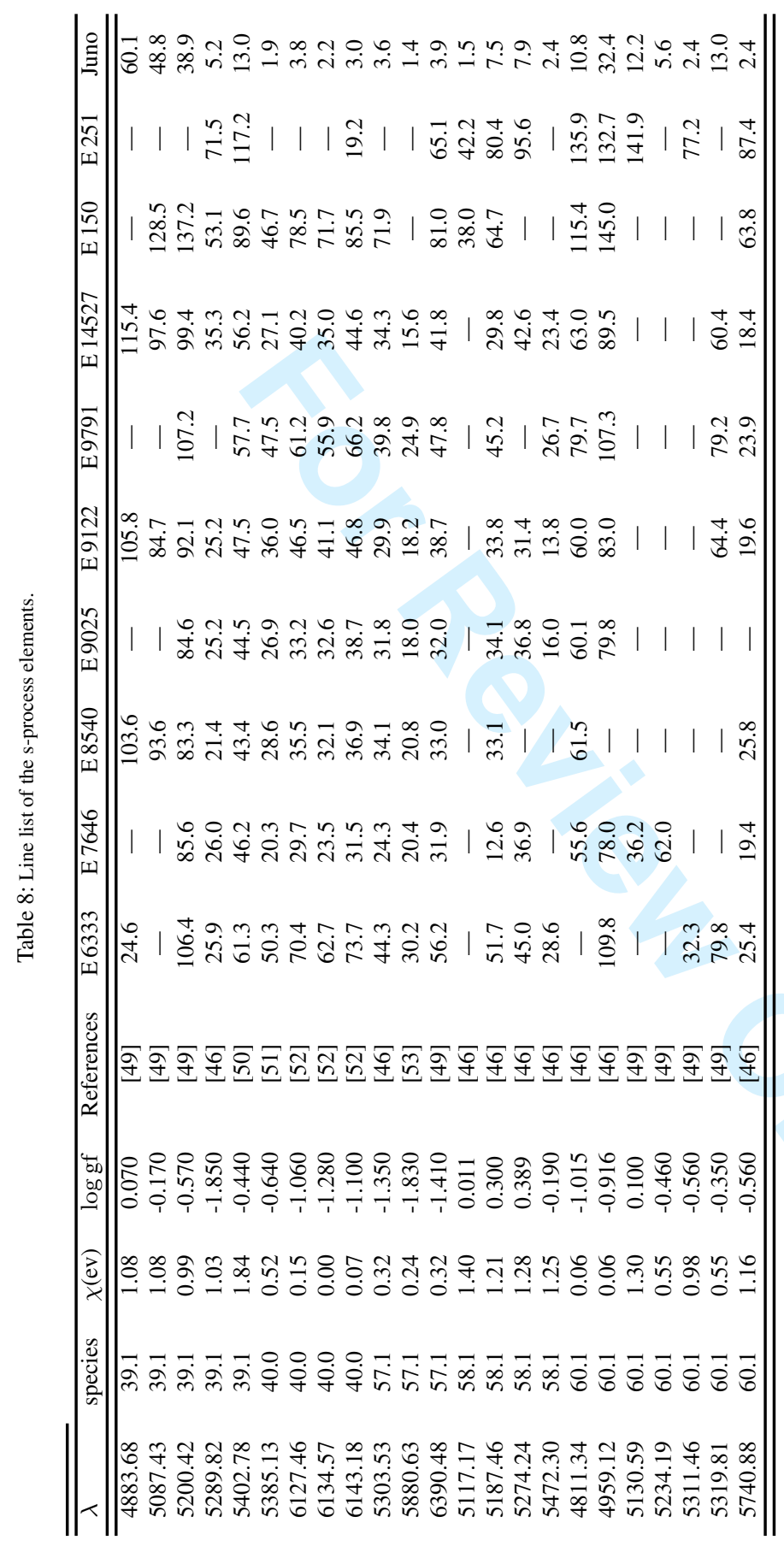

NRC Canada 